\title{
Frecuencia y características de la morbilidad genética en un hospital pediátrico
}

\author{
Rodrigo Moreno $\mathrm{S}^{1}$; Manuel Aspillaga $\mathrm{H}^{2}{ }^{2}$; Isabel Avendaño B. ${ }^{2}$ \\ Frequency and characteristics of the genetic disease \\ in a pedjatric hospital
}

\begin{abstract}
"equercy and characteristics of patients discharged with recogn zed genetic diseases by current technical literature available in 1988 (GD) from a pediatric hospital at Santiago, Chile, are described. Ori the last three months of year 1983, a prospective study on 2,987 hospital admissions corresponding to 1,196 patients was dons. I'reva. lence and incidence of $\mathrm{GD}$ were $62.5 \%$ and $17 \%$ aming hostital acmissions as a whole and $49.7 \% \%$ and $47.7 \%$ respectively when only indlvidıal patients were considered. No sex differences were found between patients with and without GD, but the trequency of GD was siunificantly higher in patients staving $7 \mathrm{cr}$ more weeks in hospital (12\% vs. 3.4\%), as among those iving required two or more admissions $(10.9 \%$ vs. $4.3 \%)$ or vere refered from outside metropolitan Santiago $(37.3 \%$ v5. $5.1 \%$ ). i irst ten more frequent $\mathrm{G} D$, in decreasing order of importance, were cardiac malformations, leukemias, cleft lip andior palate, congenital dislocation of the hip, epilepsy and convulsions, idiopathic scoliosis, Down's syndrome, anat malformations, aganglionic megacolon and cystic fibrosis. The importance of diseases with genet ic background is thus emphasized in a pediatric hosp ita!

(Key wo, ds: genetics, medical, genetic diseases, incidence, prevalence, hospitalization.)
\end{abstract}

La morbilidad genética incluye aquellas enfer. medades en que se reconoce la participación de genes, cromosomas o ambos en su determina. ción ${ }^{1}$. La etiologia genética conlleva un deteminismo móbido y de pronóstico y tratamientos crónicos o quirúrgicos cuando los hay. La pre. vención oficiente sólo es posible antes de la concepción, mediante asesoramiento genético. La importancia de las enfermcdades genéticas radica, además, en su aumento en términos relativos y absolutos ${ }^{2}, 3$, jo que ha producido cambios en el tipo de enfermedades de los pacientes ad. mitidos a los hospitales, donde se concentran los recursos humanos y técnicos requeridos para

1. Becsdo en Genética Clínica. Facultad de Medicina, Universidad de Chile.

2. Unidad de Genética. Departamento de Pediatría, Hospital Luis Caivo Mackenna. su tratamiento ${ }^{4-6}$. De estos cambios han dado cuenta publicaciones nacionales. Sin embargo, ellas son insuficientes para evaluar la magnitud del problema o prever su evolución ${ }^{7-10}$.

Este trabajo describe los resultados de un seguimiento de la morbilidad genética (MG) en pacientes pediátricos hospitalizados, para estudiar su prevalencia, incidencia, características y enfermedades más frecuentes.

\section{Pacientes y Método}

Se estudiaion 2.987 casos de hospitalizaciones, correspondientes a 1.196 pacientes indjuiduales, en el hospital Luis Calvo Mackenne desde el 30 de septiembre de 1988. Las muestras fueron obtenidas cada 7 días en 14 semanas consecutivas. Esto representa, en promedio, 213 pacientes hospitalizadus en cada tetha, lo que sc ajusta perfectanıente a las 214 camas 
ocupadas ел promedio cada día, según las estadísticas de dicluo hospital.

En cada oportunidad se realizó un catastro de los pacientes hospitalizados a la fucha en las unidades cardiovascular, cirugja, cujdados intensivos, infecciosos, lactantes, neonatología, neumología, oncología, ortopedia, quemados $y$ segunda infancia. Cada caso tue identificado por su nombre, sexo y número de ficha clínica; de esta última se obtuvo el lugar de residencia y todos los diagnósticos consignados antes y durante el periodo del estudio. La intormación flue mantentda en fjchas individuales, aunque el niño fuera rehospitalizado en periodos no consecutivos, to que permitía su seguimiento. No se incluyeron pacjentes ingresados a los servicios otorrinolaringologia, pensionado, rehabilitación y urgencia, que en promedio tienen 34 (11.4\%) de las camas en trabajo del hospital.

Los pacientes con morbjlidad genética fueron identificados como tales cuando, al thenos en uno de sus diagnósticos, se ha reconocido la participación de genes - cromosomas en su etiología de acuerdo con textos de consulta de la especialidad $3,11-13$.

Para cada una de las 14 muestras así obtenidas se estableció el total de pacientes hospitalizaóos (TPH), e) total de pacientes con morbilidad genética (TPMG) $y$, de estos úlímos, aquellos que no habían sido registrauos en anteriores oportunidades o pacientes nuevos con MG (PNMG). Estas cifras también fueron caiculadas para el total de hospitalizados y de pacientes, una vez finalizado y completado el registro de todas las muestras parciales.

La prevalencia $e$ incidencia se determinam según las siguientes fórmulas: TPMG/TPH x 100 y PNMG TPMG $\times 100$, respectivamente.
Los pacientes con MG y sin MG se compararon, en cuanto a duración de la hospitalización en seinanas, calculadas del total acumulado do pacientes de las? primeras muestras; relospitalizaciones; sexo, y residencia habitual. La comparación se realizó mediante el uso de porcentajes y las técnicas estadísticas correspondientes ${ }^{14}$. Se consideraron estadisticamente significativas (ES) diferencias con $\mathrm{p} \leqslant 0,05$.

Finalmente se expresaron en orden decreciente las 10 enfermedades genéticas más lrecuentes, según tasas por 1.000 pacjentes.

\section{Resultados}

En la tabla 1 se detaila, para cada una de las 14 muestras, el total de pacientes hospitalizados (TPH), el total de pacientes con morbilidad genética (TPMG) y los pacientes nuevos con $M G$ (PNMG), con la prevalencia e incidencia obtenidas en cada fecha y los totales de ellas, hospitalaria y por pacientes, respectivamente.

De los 2.987 pacientes hospitalizados, 1.868 presentaban al menos un diagnóstico de genéti$c 0$, que corresponde a prevalencia hospitalaria de $62,5 \%$, rango $57,2 \%$ a $67 \%$. En el período se detectaron 474 pacientes nuevos entre las muestras 2 y 14 , esto es, una incidencia hospitalaria de $17 \%$, rango $12,1 \%$ a $19,9 \%$

Tabla 1

Prevalencia e incidencia de morbilidad genética en el hospital Luis Calvo Mackenna

\begin{tabular}{|c|c|c|c|c|c|}
\hline $\begin{array}{l}\text { Muestra } \\
\text { (semana) }\end{array}$ & $\underset{n}{\text { TP. H }}$ & $\begin{array}{c}\text { TP. } M G \\
n\end{array}$ & $\begin{array}{c}\text { Preval, } \\
\%\end{array}$ & $\begin{array}{c}\text { PN. MG } \\
\text { n }\end{array}$ & $\underset{\%}{\text { Incid. }}$ \\
\hline 1 & 203 & 125 & 61,6 & - & - \\
\hline 2 & 233 & 146 & 62,7 & 45 & 19,3 \\
\hline 3 & 240 & 160 & 66.7 & 44 & 18,3 \\
\hline 4 & 235 & 148 & 63,0 & 41 & 17,4 \\
\hline$\xi$ & 230 & 141 & 61,3 & 42 & 18,3 \\
\hline 6 & 215 & 123 & 57,2 & 26 & 12,1 \\
\hline 7 & 221 & 133 & 60,2 & 39 & 17,6 \\
\hline 8 & 221 & 131 & 59,3 & 38 & 17,2 \\
\hline 9 & 236 & 146 & 61,9 & 37 & 15,7 \\
\hline 10 & 226 & 145 & 64,2 & 45 & 19,9 \\
\hline 11 & 215 & $13 ?$ & 63,7 & 32 & 14,9 \\
\hline 12 & 179 & 120 & 67,0 & 31 & 17,3 \\
\hline 13 & 165 & 107 & 64,8 & 27 & 16,4 \\
\hline 14 & 168 & 106 & 63,1 & 27 & 16,1 \\
\hline $\begin{array}{c}\text { Total } \\
\text { hospitatizados }\end{array}$ & 2.987 & 1.868 & 62,5 & 474 & 17,0 \\
\hline Pacientes & 1.196 & $\$ 95$ & 49,7 & 474 & $47,7 *$ \\
\hline
\end{tabular}

TP: total pacientes. H: hospitalizados. MG morbilidad. *: el denominador corresponde a 993 pacientes. PN: pacientes nuevos. Preval,: prevalencia. Incid.: incidencia. л: número de casos. 
Al considerar a cada niño sólo una vez en todo el período, lâs 2.987 hospitalizaciones correspon. dieron a 1.196 pacientes distintos $y$, en el caso de la morbilidad genética, a 1.868 hospitaliza. ciones para 595 nifios. Los pacientes mostraron prevalencia de $49,75 \%$ e incidencia de $47,7 \%$, considerando que se detectaron 474 PNMG entre 993 enfermos.

Lus niños de sexo masculino presentaron más morbilidad genética $(56,3 \%)$ y no genética $(58,7 \%)$ que las mujeres, diferencias que no fue. ron estad isticamente significativas.

En la tabla 2 se muestra la duración de la hospitalización en semanas, expresada en porcentaje del total acumulado de pacientes en las 7 primeras muestras. Siempre fue mayor para los pacientes con MG, con diferencias de alta significación estadística. Aún en la semana 7 habia 3 veces más pacientes con $\mathrm{MG}$ que sin ella $(12 \%$ y $3,4 \%)$. Asimismo, $60,5 \%$ de los niños sin MC egresaban antes de una semana de hospitalización, en cambio los que tenían MG sólo alcanzaban proporciones similares de egreso, $65,2 \%$, a la tercera semana de haber ingresado.

La tabla 3 describe lo ocurrido con las rehospitalizaciones. La proporción de rehospitalizados entre niños con MG (10,9\%) duplicó a la de los que reingresaron sin MG $(4,3 \%)$. Los niños con MG también fueron rehospitalizados mayor número de veces. Las diferencias son estadísticamente significativas en todos los grupos considerados, salvo el que presentó 3 reingresos.

La residencia habitual (tabla 4) de la mayoría de los pacientes era Santiago, pero la proporción de éstos con o sin MG era diferente: $60,2 \%$ y $91,2 \%$, respectivamente. En cambio entre los niños procedentes de las regiones, en su mayoría del sur del país, esta razón era inversa, con franco predominio de los pacientes con MG sobre los que no la tenían $(37,3$ vs. $5,1 \%)$. En ambos casos

Tabla 2

Pacientes hospitalizados con morbilidad genética y no genética al término de cada una de las primeras siete semanas del estudio

\begin{tabular}{|c|c|c|c|c|c|c|}
\hline \multirow[t]{2}{*}{ Semana } & \multicolumn{2}{|c|}{$\begin{array}{l}\text { Enfermedad } \\
\text { genética }\end{array}$} & \multicolumn{2}{|c|}{$\begin{array}{l}\text { Enfermedad } \\
\text { no genética }\end{array}$} & \multirow[t]{2}{*}{$\mathrm{x}$ calculado } & \multirow[t]{2}{*}{$\mathrm{p}$} \\
\hline & n & $\%$ & $\mathrm{n}$ & $\%$ & & \\
\hline NAP & 374 & 100,0 & 352 & 100,0 & & \\
\hline 1 & 239 & 63,9 & 139 & 39,5 & 6,58 & $<0,0001$ \\
\hline 2 & 174 & 46,5 & 69 & 19,6 & 7,68 & $<0,0001$ \\
\hline 3 & 130 & 34,8 & 41 & 11,6 & 7,33 & $<0,0001$ \\
\hline 4 & 98 & 26,2 & 29 & 8,2 & 6,36 & $<0,0001$ \\
\hline 5 & 71 & 19,0 & 23 & 6,5 & 4,99 & $<0,0001$ \\
\hline 6 & 59 & 15,8 & 15 & 4,3 & 5,12 & $<0,0001$ \\
\hline 7 & 45 & 12,0 & 12 & 3,4 & 5,31 & $<0,0001$ \\
\hline
\end{tabular}

NAP : rúmero acumulado de pacientes.

$\mathrm{x} \quad$ : valor del estimador estadístico para proporciones

Tabla 3

Frecuencia de rehospitalizaciones en pacientes con morbilidad genética y no genética

\begin{tabular}{|c|c|c|c|c|c|c|}
\hline \multirow[t]{2}{*}{$\begin{array}{l}\text { Número de } \\
\text { reingresos }\end{array}$} & \multicolumn{2}{|c|}{$\begin{array}{l}\text { Enfermedad genética } \\
\text { n: } 595 \text { ptes. }\end{array}$} & \multicolumn{2}{|c|}{$\begin{array}{l}\text { Enfermedad no genética } \\
\text { n: } 601 \text { ptes. }\end{array}$} & \multirow[t]{2}{*}{ x cajculado } & \multirow[t]{2}{*}{$\mathbf{p}$} \\
\hline & $\mathbf{n}$ & $\%$ & n & \% & & \\
\hline $\begin{array}{l}1 \\
2 \\
3\end{array}$ & $\begin{array}{r}55 \\
9 \\
1\end{array}$ & $\begin{array}{l}9,24 \\
1,51 \\
0,16\end{array}$ & $\begin{array}{r}25 \\
1 \\
0\end{array}$ & $\begin{array}{l}4,15 \\
0,16 \\
0,00\end{array}$ & $\begin{array}{l}3,51 \\
2,55 \\
1,00\end{array}$ & $\begin{array}{c}<\quad 0,02 \\
<\quad 0,02 \\
\text { NS }\end{array}$ \\
\hline Total & 65 & 10,92 & 26 & 4,32 & 4,30 & $<0,0001$ \\
\hline
\end{tabular}

$\mathrm{x}$ : valor del cstimador estadístico para proporciones. 
Tabla 4

Residencia habitual de los pacientes con tipos de morbilidad genética y no genética

\begin{tabular}{|c|c|c|c|c|c|c|}
\hline \multirow[t]{2}{*}{ Residencia } & \multicolumn{2}{|c|}{ Enfermedad genética } & \multicolumn{2}{|c|}{ Enfermedad no genétjca } & \multirow[b]{2}{*}{$\mathrm{x}$ calculado } & \multirow[b]{2}{*}{$\mathrm{p}$} \\
\hline & $\mathbf{n}$ & $\%$ & $\mathrm{n}$ & $\%$ & & \\
\hline Santiago & 358 & 60,2 & 548 & 91,2 & 12,53 & $<0,0001$ \\
\hline Regiones & 222 & 37.3 & 31 & 5,1 & 13,53 & $<0,0001$ \\
\hline S/informe & 15 & 2,5 & 22 & 3.7 & 1,13 & $\mathrm{NS}$ \\
\hline Total & 595 & 100,0 & 601 & 100,0 & & \\
\hline
\end{tabular}

x: valor del estinador estadístico para proporciones.

las diferencias eran significativas. En los pacientes sin información de procedencia los porcentajes de uno y otro tipo de morbilidad eran parecidos: se trataba de pacientes de la Casa Nacional del Nifio, centros de alimentación de la Corporación para la Nutrición Infantil (CONDN) o referidos de otros hospitales.

En la tabla 5 se despliegan las 10 causas de morbilidad genética más frecuentes encontradas en este estudjo, expresadas en tasas por 1.000 pacientes: las malformaciones cardiacas predominaban ampliamente $(123,7$ por mil), seguidas por las leucemias $(24,2$ por mil). En último lu. gar estaban el megacolon agangliónico y la fibrosis quistica $(9,4$ por mil cada una $)$.

\section{Discusión}

Los estudios de prevalencia e incidencia de morbilidad hospitalaria constituyen desafios en nuestro medio, debido a que no existen registros completos y de fácil acceso, como serían los

Tabla 5

Morbilidad genétjca más frecuente en pacjentes del hospital Luis Calvo Mackenna

\begin{tabular}{lc}
\hline Morbilidad genética & Tasa x 1.000 pacientes \\
\hline Malformaciones cardiacas & 123,7 \\
Lelicemias & 24,2 \\
Fisuras labiopalatinas & 20,1 \\
Displasia congénita de caderas & 17,6 \\
Epilepsia y convulsiones & 16,7 \\
Escoliosis & 16,7 \\
Sindrome de Down & 15,0 \\
Malfomnaciones anales & 12,5 \\
Megacolon agangliónico & 9,4 \\
Fibrosis quística & 9,4 \\
\hline
\end{tabular}

computacionales ${ }^{4,15}$. Sin embargo, cuando son posibles, ofrecen ciertas ventajas, como diagnósticos más precisos, que a su vez constituyen estimadores estadísticos más reales, permitiendo precisar mejor las demandas asociadas de recur. sos.

E1 intervalo semanal de muestreo es razonable para el seguimiento de pacientes hospitalizados, pues registra $99,5 \%$ de los casos hospitalizados en cada fecha. Aunque algunos niños pueden egresar y reingresar en dicho lapso y de este modo ser considerados como permaneciendo hospitalizados, ellos representan excepciones que no invalidan los resultados.

Al corregir la prevalencia y la incidencia por pacientes individuales se obtiene mejor la estimación de la frecuencia de la morbilidad genética, evitando la inclusión reiterada de niños con hospitalizaciones prolongadas que exageran la prevalencia y reducen erróneaniente la incidencia.

La exclusión de todos los pacientes de los servicios de rapido egreso, en este estudio, dejó de lado a $11,4 \%$ de los niños hospitalizados en el periodo, pero no habiéndose hecho selección alguna con ellos, es posible que no se introduzcan por este motivo sesgos sistemáticos en los resultados.

No se conoce en Chile la prevalencia hospitalaria pediátrica de MG. Nuestras cifras concuerdan con lo descrito en otros paíse ${ }^{17-19}$. $\mathrm{La}$ incidencia de enfermedades genéticas es mayor que la encontrada en otro hospital infantil de Santiago de similar complejidad ${ }^{9}$ y en el servicio de lactantes del Hospital Regional de Temuco $^{16}$. Esas diferencias con otros estudios nacionales se deben en parte a los distintos méto$\operatorname{dos}^{15}$ y complejidades asistenciales consideradas ${ }^{15}$. Con respecto a los resultados obtenidos un hospital de similar complejidad ${ }^{15}$, nuestras cifras son más altas porque en el estudio men- 
cionado no fueron considerados todos los diagnósticos de cada uno de los pacientes $y$, en el momento en que se hizo dicho trabajo, aún no se reconocia el sustrato genético de varias de las enfermedades incluidas por nosotros ${ }^{3,11-13}$. Actualmente se acepta, en general, que las afecciones no genéticas son las asociadas exclusivamente a factores ambientales, como accidentes, violencia, infecciones y problemas nutricionales carenciales ${ }^{19}$.

La escasa variación de la prevalencia e incidencia de la MG permite suponer que será cons. tante a lo largo del año, luego se puede esperar que entre niños hospitalizados 2 de cada 3 y en ingresos nuevos 1 de cada 2 , tengan como diag. nóstico una enfermedad de origen genético. Esto permitiría programar las acciones necesarias para resolver problemas de hospitalización prolonga$\mathrm{da}$, rehospitalizaciones y derivación desde otras regiones asociadas a pacientes con MG. La soluciốn para los pacientes de otras regiones podría ser dotar a los hospitales respectivos con la capacidad para diagnosticar y manejar la $M G$ con mayor relevancia local.

E) hallazgo de mayor frecuencia de morbilidad en el sexo masculino coincide con otras descripciones sobre la materia ${ }^{7}, 10,16$.

Las enfermedades más frecuentes en nuestra casuística fueron las malformaciones cardíacas, con tasas mucho mayores que las estimadas para recién nacidos en Santiago por otros autores. Ello se debe en alguna medida a que la unidad cardiovascular de este hospital es uno de los centros médico-quirúrgicos cardiológicos de referencia deJ pais. Incluyendo nuestra casuistica, las demás estimaciones tambiên reúnen las malformaciones cardiacas bajo una sola entidad ${ }^{10,20}$, aunque corresponden a distintos tipos de determinación genética.

Las frecuencias de fisuras labiopalatinas, de displasia congénita de caderas, síndrome de Down y malformaciones anales, de este estudio, son siete o más veces más altas que las descritas en recién nacidos ${ }^{10}$, lo cual confirma el impacto de la MG entre las causas de hospitalización.

El aumento en la morbilidad genética hospitalaria pediátrica debería reflejarse también en los servicios de adultos ${ }^{21}$, debido a la evolución crónica de las enfermedades genéticas y a la mayor sobrevida de estos pacientes como efecto de la disminución en la mortalidad infantil ${ }^{22}$, en la cual la morbilidad genética tiene mayor incidencia ${ }^{9,23}$.

\section{Resumen}

Se describen la frecuencia y características de pacientes con morbilidad genética (MG) en un hospital pediátrico de Santiago, Chile. EJ último trimestre de 1988 se realizó un seguimiento de 2.987 casos hospitalizados que correspondieron a 1.196 pacientes. La prevalencia y la incidencia medidas por ingresos hospitalarios fueron $62,5 \%$ y $17 \%$ y por pacientes individuales $49,75 \%$ y $47,7 \%$, respectivamente. Los pacientes con y sin MG no difirieron en el sexo, pero los niños con MG fueron significativamente más frecuentes que los sin MG entre los que permanecian hospitalizados por 7 semanas (12\% vs. $3,4 \%$ ); sufrian rehospitalizaciones $(10,9 \%$ vs. $4,3 \%) \circ$ eran residentes de otras regiones $(37,3 \%$ vs. $5,1 \%$ ). Las diez primeras $M G$, por frecuencia, fueron las malfonmaciones cardíacas; leucemias; fisuras de labiopalatinas, displasia congénita de caderas; epilepsia y convulsiones; escoliosis; sindrome de Down; malformaciones anales; megacolon agangliónico y fibrosis quîstica. Nuestros resultados evidencian la creciente importancia de las enfermedades de tiología genética entre los niños hospitalizados.

(Palabras clave: genética, médica, enfermeda. des genéticas, incidencia, prevalencia, hospitalización.)

\section{Agradecimientos}

Al personal de los servicjos y al servicio de estad istica y archivo del hospital Luis Calvo Mackenna, por su colaboración. Al personal de la Unidad de Genética, por su constante estímulo.

\section{Referencias}

1. Mackusick, V.: The morbid anatomy of the human genome: A review of gene mapping in clinical medicine. Part I. Medicine 1986;65:1-33.

2. Cruz-Coke. R.: Principios genéticos en salud pública. En: Meneghello, J. ed. Pedjatria, Santiago: Editorial Mediterráneo $1985 ; 1209-1213$.

3. MacKusick, $V .:$ Mendelian inheritance in man. Catalogs of autosomal dominant, autosomal recessive, and $\mathrm{X}$-linked phenotypes. Baltimore: The Johns Hopkins University Press 1988.

4. Weldt, E.: Hering, E.- Estudio computarizado de morbilidad y mortalidad en recién racidos. Rev Clijl Pediatr 1984; 55: 322-330.

5. Nozer, J.; Castillo, S,; Cifuentes, L.; Ruiz, G.: Pizarro, $T_{.}$: Parado, $L_{\text {. }}$ : Frecuencia de malforma. ciones congénitas en Chile en el período 1969 . 1986. Resultados de un estudio colaborativo latinoamericano. Rev Méd Chile 1989; 117: 219-227. 
6. Nazer, J.; Díaz, G.: Pizarro, M.: Malfomaciones congénitas I. Estudio clínico y epidemiológico. Pediatría (Santiago), 1978; $21: 295-303$.

7. Cruz-Coke, R.; Volenzuela, C.; Navorro, I.: La morbidité génétique: méthodes de mesures et résultats obtenus a Santiago. Population 1972;6; 1045-1052.

B. Cruz-Coke, $R$; Volenzuela, $C$. Hercditary diseases and congenital anomalies in the population of Santiago, Chile, A preliminary communication: Birth Defects: Original article series 1974; 10 (10): 226-229.

9. Cruz-Coke, R.; Valenzuela, C.: Enfermedades genéticas en Chile Rev Méd Chile 1975; 103: $327-330$.

10. Nazer, $J:$ Dáaz, G.; Pizarro, M.: Estudio clínico y epidemiológjco de las malformaciones congénitas. II Incidencia de malformaciones congénitas en el área norte de Santiago. Pediatría (Santiago) 1979; $22: 70-76$

11. Bergsma, $D .:$ Birth defects compendium, New York: Published for The National Foundation. March of Dimes, 1979

12. De Grouchy, J.; Turleau, C.: Clinical atlas of human chromosomes, New York: John Wiley \& Sons, 1977

13. Emery, A.; Rimoin, D. Principles and practice of medical genetics, Churchill Livingstone, Edinburgh, London, Melbourne and New York, 1983.

14. Emery, $\boldsymbol{A}$ : Methodology in medical genetics, Churchill Livingstone, Kdinburgh, London and New York, 1976.
15. Cruz-Coke, R.; Valenzuela, C.; Kojchen, J.: Programa de monitoría y vigitancia de salud mediante un macrosistema de informática. Rev Méd Chile $1973 ; 101: 490-498$.

16. Moreno, R.i Navarrete, P.; Aros, C: Detección clínica de enfermedades genéticas en lactantes hospitalizados. Hospital Regional de Temuco. Resumen presentado XXIII Reutión Anual Sociedad de Genética de Chile, 23 al 25 de agosto 1990 , Valparaiso, Chile.

17. Miller, J.: Human genetics in public health research and programming. En: Schacht, E.: Human Genetics in Public Health, Minnesota, Dept. of Health, Minneapolis, 1964.

18. Day, N.: Holmes, L.B.: The incidence of genetic disease in a university hospital population. Am J Hum Genet 1973;25: 237-246.

19. Hall, J.G.: Powers, E.K.; Mcllvaine, R.T.: Ean. V.H.: The frequency and financial burden of genetic disease in a pediatric hospital. Am J Med Genet 1978: 1:417436

20. Philippi, O.; Rencoret, G.; Pinto. M. ef al.: Incidencia de cardiopatias congénitas en nacidos vivos. Rev Chil Pediatr 1986;57: 447-451.

21. Cruz.Coke, R.: Valenzuela. C: Enfermedades hercditarias en un hospital general. Rev Med Chile $1973 ; 101: 212+215$.

22. Mardones. G.; Castillo, B.: Solts. F.: Evolución de la mortalidad por causas $(1960 \cdot 1982)$. Rev Méd Chile 1989-117:821-828.

23. Armendares, S.: Cortés, $R$, De la Rosa, 1.: El componente genético en la mortalidad infantil. Rev Invest Clin (Méx.), 1974; 26: 3-18.

Esta publicación está disponible en copias de microfilms de 16 y $35 \mathrm{~mm}$ y microfichas de $105 \mathrm{~mm}$, las que pueden solicitarse a:

University Microfilms International

300 North Zeeb Road

Ann Arbor, Michigan 48106, USA.

This joumal is also available in $16 \mathrm{~mm}$ microfilm, $35 \mathrm{~mm}$ microfilm and $105 \mathrm{~mm}$ microfilm copies through University Microfilms International, 300 North Zeeb Road, Ann Arbor, Michigan 48106, USA. 